\title{
The VIL (Virtual Immersive Learning) Test-Bed
}

\author{
(Invited paper - Special session: Design of communication system architectures for mobile virtual immersive services) \\ Franco Davoli \\ National Inter-University \\ Consortium for \\ Telecommunications (CNIT) \\ Via Opera Pia 13, \\ I-16145 Genova, Italy \\ +39010 3532732 \\ franco.davoli@cnit.it \\ Gianluca Massei \\ National Inter-University \\ Consortium for \\ Telecommunications (CNIT) \\ Via Diocleziano 328, \\ I-80125 Naples, Italy \\ +390812303311 \\ gianluca.massei@cnit.it \\ Amedeo Scarpiello \\ National Inter-University \\ Consortium for \\ Telecommunications (CNIT) \\ Via Diocleziano 328, \\ I-80125 Naples, Italy \\ +390812303311 \\ amedeo.scarpiello@cnit.it \\ Sandro Zappatore \\ National Inter-University \\ Consortium for \\ Telecommunications (CNIT) \\ Via Opera Pia 13, \\ I-16145 Genova, Italy \\ +390103532990 \\ sandro.zappatore@cnit.it
}

\begin{abstract}
The Virtual Immersive Learning (VIL) test-bed implements a virtual collaborative immersive environment, capable of integrating natural contexts and typical gestures, which may occur during traditional lectures, enhanced with advanced experimental sessions. The main aim of the paper is to describe the test-bed motivations, as well as the most significant choices, both hardware and software, adopted for its implementation. The novelty of the authors' approach essentially relies on its capability of remarking and emphasizing results that are the output of the national project VICOM, and "putting the pieces together" in a well-integrated framework. These features, along with its high portability, good flexibility, and, above all, low cost, make this approach appropriate for educational and training purposes, mainly concerning measurements on telecommunication systems, at universities and research centers, as well as enterprises. Moreover, the methodology can be employed for remote access to and sharing of costly measurement equipment in many different fields of activity.
\end{abstract}

\section{Categories and Subject Descriptors}

H.5.1 [Information Interfaces and Presentation]: Multimedia Information Systems - Artificial, augmented, and virtual realities. H.5.2 [Information Interfaces and Presentation]: User Interfaces - Graphical user interface (GUI), haptic I/O, input devices and strategies, interaction style

H.5.3 [Information Interfaces and Presentation]: Group and Organization Interfaces - Computer-Supported cooperative work K..3.1 [Computers and Education]: Computer Uses in Education - Collaborative learning, distance learning

\section{General Terms}

Measurement, Design, Experimentation.

\section{Keywords}

Distance learning, Remote Measurement, Virtual Reality, Scene Analysis, Cooperative work, Graphical User Interface, Context Awareness, Immersive interaction.

Permission to make digital or hard copies of all or part of this work for personal or classroom use is granted without fee provided that copies are not made or distributed for profit or commercial advantage and that copies bear this notice and the full citation on the first page. To copy otherwise, to republish, to post on servers or to redistribute to lists, requires prior specific permission and/or a fee. IMMERSCOM 2007, October 10-12, Verona, Italy

Copyright () 2007 978-963-9799-06-6

DOI 10.4108/ICST.IMMERSCOM2007.2110

\section{INTRODUCTION}

VICOM (Virtual Immersive Communications) is a national project funded by the Italian Ministry of Education, University and Research (MIUR), started in November 2002 and ended in May 2006 (http://www.vicom-project.it). The project goal has been the design of a communication system's architecture able to provide mobile virtual immersive services. The architecture effectiveness is proved with two service test-beds, denoted as Mobility in Immersive Environment (MIE) and Virtual Immersive Learning (VIL), respectively. In particular, the VIL test-bed implements a virtual collaborative immersive environment, capable of integrating natural contexts and typical gestures, which may occur during traditional lectures, enhanced with advanced experimental sessions. Two training courses are realized: the first one is oriented to virtual restoration of paintings, whereas the second one concerns e-measurement applications, where students are able to remotely control real devices and instrumentation, located at the National Laboratory for Multimedia Communications in Naples, Italy, and at WiLab in Bologna, Italy.

A 3D virtual reality application allows the real time interaction between a lecturer or instructor and st udents, who are not physically present in the same classroom. Students are grouped inside a number of well-equipped classrooms, which are interconnected by an IP network.

Traditional approaches to Virtual Reality (VR) are based on

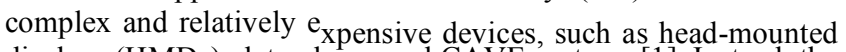
displays (HMDs), data gloves and CAVE systems [1]. Instead, the proposed approach to realize the VIL test_bed emphasizes results that are the output of research activities related to other work packages of the VICOM p roject. In particular, it exploits audio and video processing algorithms to realize an immersive interaction with the virtual class, a specific database to share and manage all context information, a multimedia board and an embedded haptic interface to $\mathrm{sho}_{\mathrm{W}}$ different approaches to virtual reality applications, hardware/software architectures specifically designed and realized to control real measurement instruments and devices, and virtual restoration tools to improve the quality of the digital reproductions.

\section{HARDWARE COMPONENTS}

In the VIL scenario the generic user reaches a VIL real classroom and logs in to the system through an accounting phase, to define the user's profile. Then, the lecturer and students enter the virtual classroom, where they are represented by their avatars, and reach their own virtual workspace. So, the real-time lecture takes place 
in a virtual context-aware environment, where interactions occur in a natural way, by means of scene analysis systems and immersive input devices. Finally lectures are complemented with laboratory experiences, oriented to Supervised Tele-Restoration and Cooperative Tele-Measurements, exploiting specialized virtual laboratory software.

The proposed scenario has been realized in order to be compliant with the economical resources of the VICOM project. To this aim, all useful research results from other work packages have been emphasized, rather than buying very expensive hardware available on the market.

The fulfillment of the VIL test-bed's goals has required the specification and the acquisition of the equipment of some enhanced classrooms, through which lecturer and students can take part in the immersive lecture. These classrooms are interconnected through the CNIT national network, mainly based on a satellite platform, allowing the bidirectional interconnection of a large part of CNIT research units and laboratories. The network operates in Skyplex technology over the $\mathrm{Ka}$ band (HotBird6 Satellite), and provides an overall satellite bandwidth of $2 \mathrm{Mbps}$, shared among the active earth stations.

Since different types of enhanced classrooms are possible, each center can choose the test-bed components to highlight. A fully equipped classroom includes the hardware components explained in the following, to remark all significant aspects of the test-bed:

- Video rendering systems. For the students' class we have selected a visualization system composed by a projection screen, two linear polarization filters, two XGA projectors and passive glasses (see Figure 1). An auto-stereoscopic display is used for the lecturer. Both systems must be equipped with a professional graphics workstation.

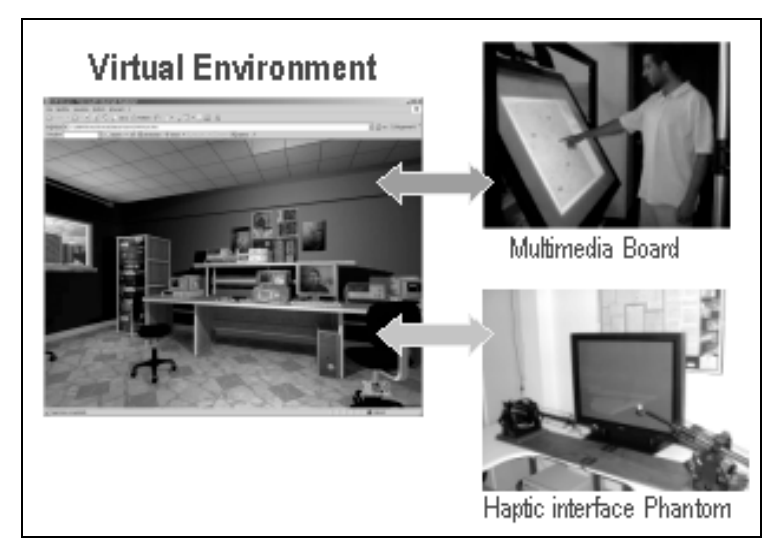

Figure 1: Video rendering system for the students' class

- Audio rendering systems. For the students' class we have chosen wireless head-phones, while normal loudspeakers are sufficient for the lecturer.

- Input devices (see Figure 2). Any user can interact with the GUI (Graphical User Interface) through input devices providing different immersion sensations. The user can choose a simple mouse, a 3D mouse with six degrees of freedom, a haptic interface (provided by the PERCRO laboratories of Pontedera, Italy), or the Multimedia Board (provided by the CNIT research unit at the University of Florence, Italy).

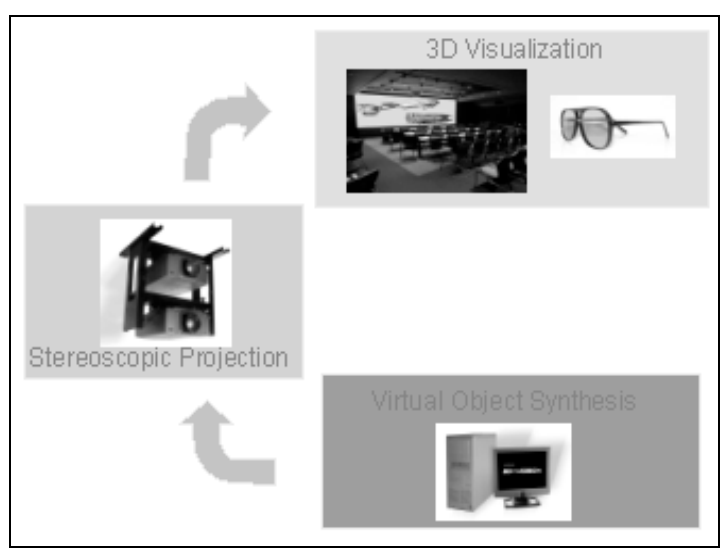

Figure 2: Input devices

- $\quad$ Contribution devices. During the lectures or the laboratory experiences, audio and video interaction of any user must be allowed. For the students' class we have selected a PTZ (Pan-Tilt-Zoom) dome camera (whose control is allowed via VISCA $^{1}$ commands) and omni-directional microphones, while simple commercial devices are sufficient for the lecturer.

- Scene Analysis systems. These systems allow the acquisition and analysis of context information. They need an accurate tuning to overcome the environment problems (room size, light, noise level, reverberation, etc...). In particular, the Audio-Location System, provided by the research unit at the Technical University of Milan, allows locating the position of the speaker making a reservation, through the phase processing of the acquired audio signals (it includes an array of microphones, audio mixer, computer for the processing, and deadening panels), while the Request Identification System, provided by the CNIT research unit at the University of Genoa, allows making a reservation for a question or intervention simply by raising a hand, by means of video processing techniques (it includes dome camera and a computer for processing). Finally, a specific application, developed by the CNIT research unit at the University of Cagliari, is able to control the PTZ dome camera to transmit the video of the student making a reservation.

\section{SOFTWARE ARCHITECTURE}

The proposed software architecture is illustrated in Figure 3. The Common Experience Manager (CEM) is certainly the main block of such architecture, as it manages both e-learning and experimental laboratory sessions. Context is captured and analyzed by the Scene Analysis (SA) module, through arrays of

\footnotetext{
1 VISCA (Video System Control Architecture) is a network protocol designed to interface a wide variety of video equipment to a computer.
} 
microphones and cameras. Such information is stored in the VIL Database and managed by a java interface.

Any student can select a synchronous or asynchronous instruction course. In the former case, the CEM manages the interaction between students and lecturer through a token-based mechanism: the lecturer is able to entirely release or to share its privileges, communicating with the CEM through an immersive GUI. Interactive Inputs (II) allow interaction with the virtual environment, while Contribution Inputs (CI) allow to ask questions during a lecture after being enabled by the lecturer: interventions occur by video and audio streaming. In the latter case, the Offline Lectures Manager (OLM) module represents the interface to enter off-line contents.

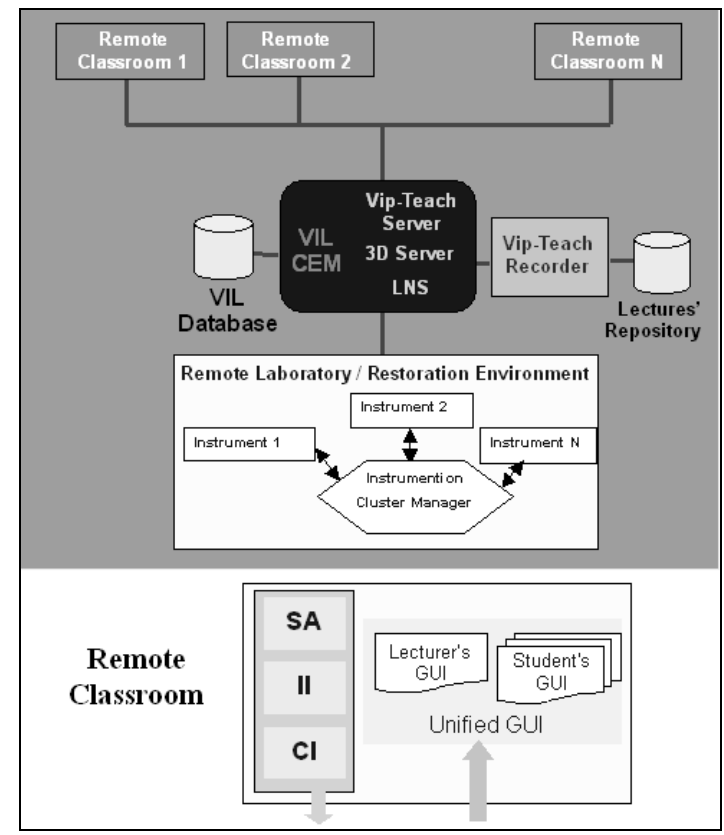

Figure 3: Main blocks of the software architecture

\subsection{Graphical User Interfaces}

A new immersive Graphical User Interface (GUI) has been developed to support 3D contents in the synchronous e-learning application Vip-Teach, provided by Lightcomm (http://www.lightcomm.it). The components of this GUI (see Figure 4) are: video (MPEG4 codec), chat, ppt presentations, 3D space, and management window (with the list of students on-line and of those making a reservation). In particular, 3D contents in the lecture session are realized in VRML (Virtual Reality Modeling Language) and controlled by Java applications [2]. They allow navigation in the $3 \mathrm{D}$ environment, management of collisions among 3D objects, visualization of the avatars of other users moving in the environment, visualization of reservation events and information about users, search of an avatar by name and selection of a laboratory session.

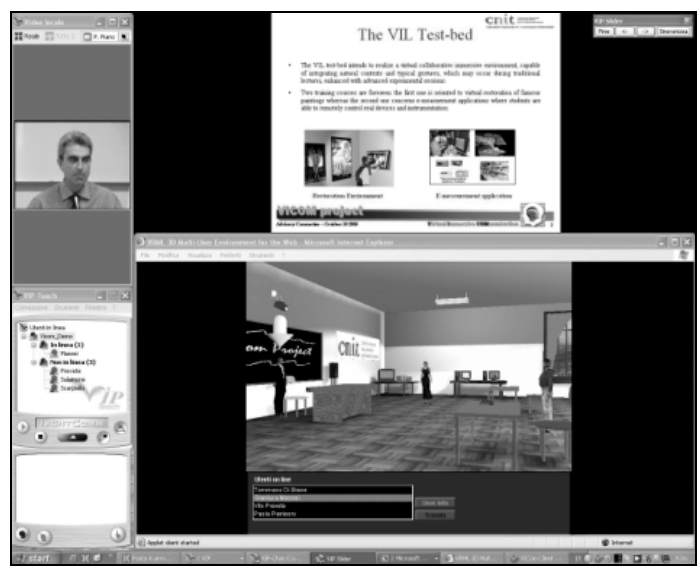

Figure 4: GUI in the lecture session

During the lecture, the lecturer can select a laboratory session, simply by clicking on a virtual door present in the scene. 3D contents in the laboratory sessions (see Figure 5) are modeled through 3D Studio Max and controlled through eXtreme Virtual Reality $(\mathrm{XVR})^{2}$ by VRMedia (http://www.vrmedia.it).

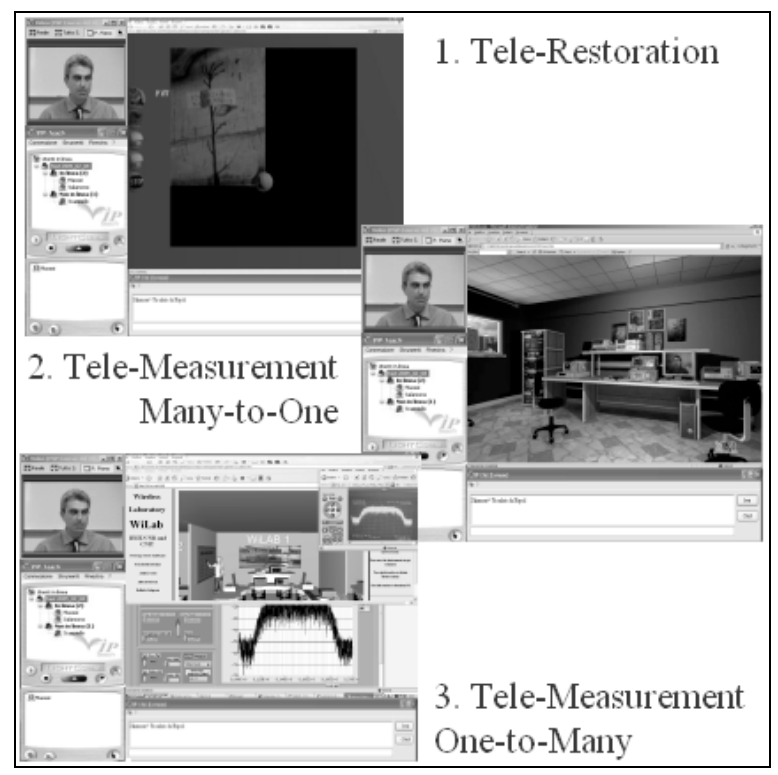

Figure 5: GUI in the laboratory sessions

The Tele-Restoration session, realized by the CNIT research units at the Universities of Florence and Pisa S. Anna, allows experimenting virtual restoration techniques (such as crack removal and lacuna filling) on high-resolution digital copies of famous paintings, while the two Tele-Measurement sessions permit to interact with real instrumentation. In the Many-to-One paradigm, developed in the CNIT National Laboratory for Multimedia Communications (Naples), the experience is

${ }^{2} \mathrm{XVR}$ is a new free technology to develop advanced multimedia content for web pages, mainly focused on 3D graphics \& sound. 
collaborative, namely the GUI interface allows the lecturer to transfer the experiment's control to the students, while in the Oneto-Many paradigm, realized in the WiLab laboratories (Bologna), it is possible to interact with a "measurement chain", whose instrumentation is geographically distributed in different locations.

\subsection{Server-side architecture}

The main components of the CEM are: the VIP-Teach Server, the LabNet Server and the 3D Server, as shown in Figure 6.

The Vip-Teach Server is able to manage users' accounts and permissions, enrol the students in the lectures and activate the PowerPoint viewer on the remote PCs. It can be followed by a web portal, for the management of the lectures' calendar and for the off-line diffusion of ppt presentations, and by a Recorder that allows recording the lecture.

The LabNet Server [3] manages access to a generic experience, guaranteeing the interoperability and the synchronization among users. Particularly, owing to a Control module, it makes the experience collaborative, allowing a super-user (the lecturer) the possibility to pass the instrumentation control (token) to users of inferior level (the students), through the VIP-Teach client interface. Besides, owing to the Data Providing module, the instrument data are distributed to users in multicast fashion, and can be visualized on the 3D interface, via a java-based Adaptation Layer.

The $3 D$ Manager (i.e., the main component of the $3 D$ Server) is a pure Java application able to manage the VIL database and information related to the graphical representation, and handle authorizations of avatars and the logical structure of the scene.

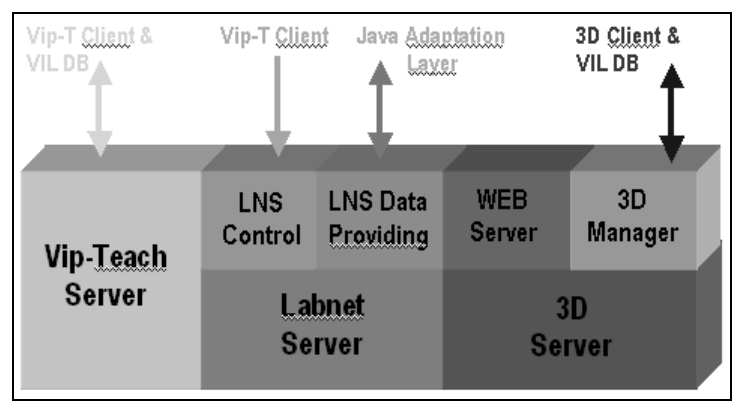

Figure 6: Main components of CEM

The software architecture for e-measurement experiences, developed at the National Laboratory for Multimedia Communications in Naples, is in shortly explained, by using a top-down approach, in Figure 7. The SW modules involved in the architecture are explained in the following.

- The 3D GUI communicates with the remaining architecture via a Java-based interface.

- The LNS (LabNet Server) manages the access of users to the experiments.

- The Experience Manager manages the allocation of the instruments in the individual experiments, the correspondence among the experiment's variables and actions on the instrumentation drivers.

- The Experience database contains the experiment table (to list the instruments involved in each one) and instrument table (to define the allocation state).

- Test-bed is the set of instrumentation drivers for emeasurement sessions.

In particular, to call the driver procedures, the Experience Manager uses Remote Procedure Calls (RPC) through SOAP (Simple Object Access Protocol) on TCP/IP [4]. The drivers recognize SOAP messages and translate them into reading/writing commands on the instruments involved in the experiment.

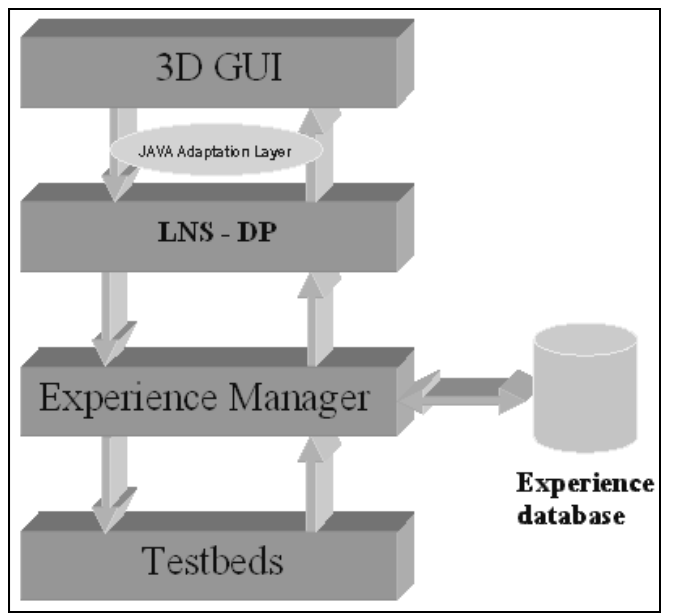

Figure 7: SW architecture for e-measurement session

\subsection{Client-side architecture}

Figure 8 shows the main components of the Remote Classroom. In accordance with the GUI, we have considered two main software modules: the VIP-Teach Client and the $3 D$ Client.

The VIP-Teach Client is able to interact with VIP-Teach Server to manage users' accounts, receive/transmit the audio and video contents from/to own peers, according to the relative roles, transfer the information related for token management to the LNS Control module, and interact with the VIL Database to publish the token holder in the context space and to extract the reservation data.

The VRML/XVR-based 3D Client must interact instead with the 3D Manager to log the users and present context information (i.e., user identity, avatar position, students in reservation), with the LNS Data Providing module to write and read instrument and painting data via the java-based adaptation layer, and with the VIL Database for data upload/download. 


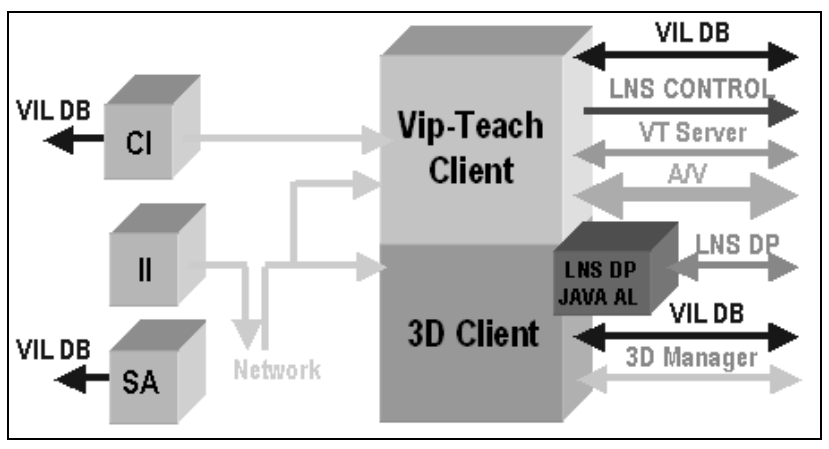

Figure 8: Main components of the Remote Classroom

\subsection{Context data exchanging}

A MySQL DB, named VIL Database and shown in Figure 9, is used to exchange context data.

It consists of 8 tables, regarding both user and environment:

- the static tables contain user profiles, authorization, environment settings and experiments' descriptions.

- the graphical data update is provided by two dynamic tables: user dialog (in which any client writes its own data) and user information (in which the 3D Manager inserts global data to provide the updates to all clients);

- $\quad$ the Hand UP table is used by external applications, such as the Scene Analysis systems and Vip-Teach, to manage the reservation;

- the location table is used in order to identify the actual experiment or to change it.

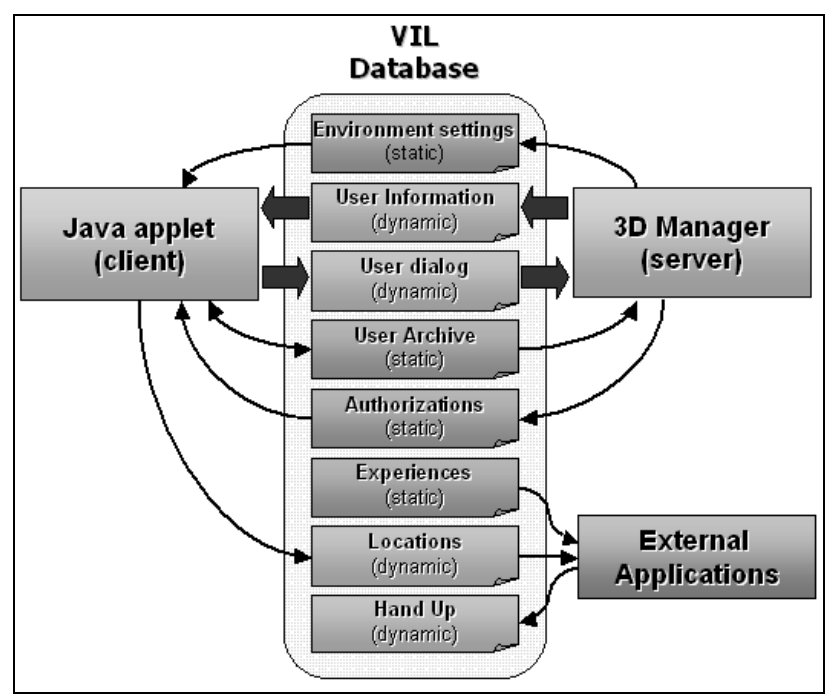

Figure 9: The VIL Database

\section{A SPECIFIC DEMO}

A specific remotely controlled demo has been set up in the Manyto-One Tele-Measurement session by the National Laboratory for Multimedia Communications in Naples. Its goal is to test the operating conditions of a WLAN, in the presence of an adjacent interfering channel, produced by a vector signal generator.

In particular, it allows the qualitative analysis of the channel throughput, by observing the quality of a received video sequence and the number of dropped packets and, at the same time, by viewing the resulting waveform on the display of a virtual instrument, representing a remotely controlled real spectrum analyzer.

As shown in Figure 10, the network segment under examination is that of a single Access Point (Cisco Bridge 350), while the interfering signal is created by an Agilent E-4438C vector signal generator. The resulting sum traverses a splitter, where a part of the signal power is directed to another Access Point, to be then transmitted over a satellite link from the National Laboratory for Multimedia Communications in Naples to any remote site; another part reaches a Spectrum Analyzer (Agilent E-4404B), where the interference phenomenon can be visualized.

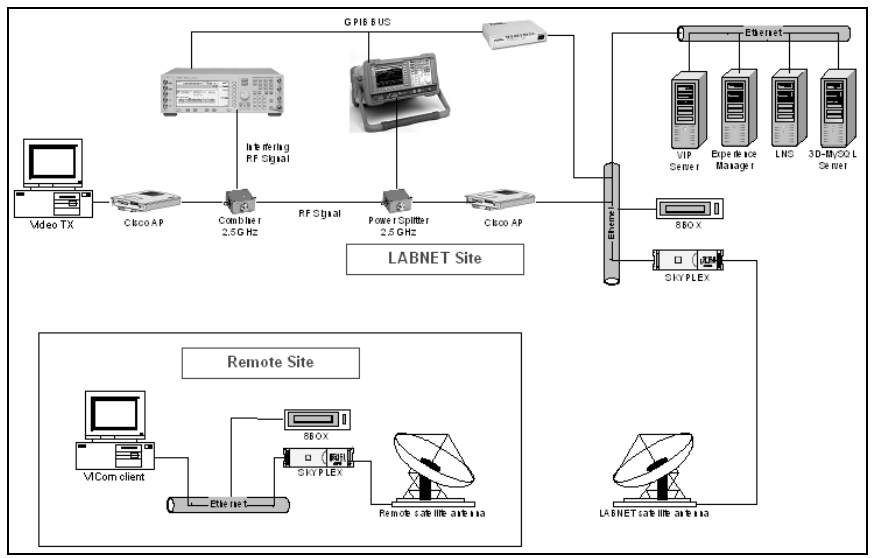

Figure 10: Demo architecture in the Many-to-One TeleMeasurement session

The signal under test is MotionJPEG-encoded video, generated by a multicast source, while the interfering traffic is a deterministic constant bit rate signal.

By using the 3D GUI (see Figure 11), it is possible to see the signal waveform on a device's display (e.g., a spectrum analyzer), to observe the quality of a received video sequence, to pass and revoke the token to/from a student, to know the statistics of a video transmission and to set the values of experiment variables, by clicking on the instrument's buttons.

For example, when the two transmissions are on non-overlapping channels (interfering traffic on $\mathrm{CH} 1$ and video one on $\mathrm{CH} 7$ ) there are no dropped packets. If the interfering signal is shifted on an adjacent channel ( $\mathrm{CH} 6)$, it is possible to see some dropped packets and a low video quality. If the two transmissions are on the same channel $(\mathrm{CH} \mathrm{7)}$, the video transmission is completely stopped. At this point, if the amplitude of the interfering signal is lowered, the video transmission can start again. 


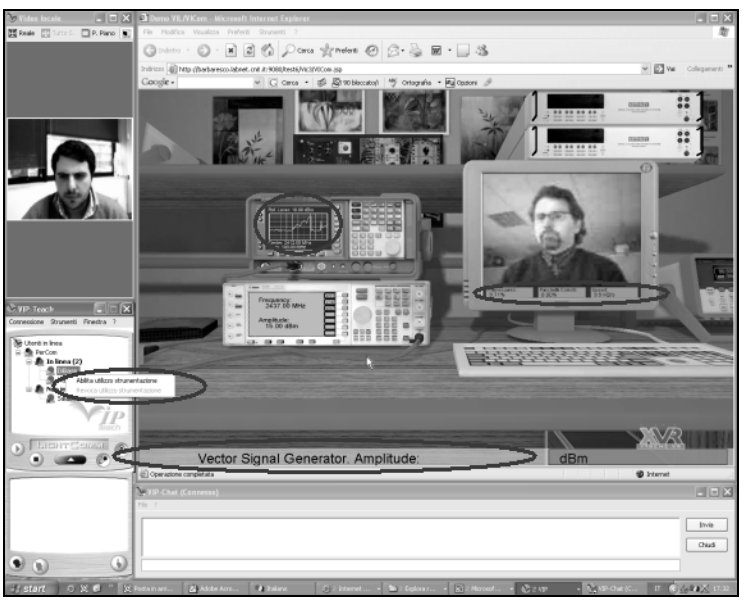

Figure 11: GUI in the Many-to-One Tele-Measurement session

\section{USER MOBILITY ISSUES}

The VIL test-bed does not address mobility issues explicitly. As a matter of fact, the core of the distance learning application does not change, even in case the client used to follow a lecture or access a laboratory session is characterized by a certain degree of mobility. Wireless access, a requisite for mobility, has been indeed considered, since the connection in the example experiment we have described relied upon a satellite link. In this respect, it is worth mentioning that the LABNET server protocol, adopted for the management of the client population in the access and control of the measurement devices, has been shown to exhibit a very satisfactory degree of robustness when used over high bandwidth-delay product networks (e.g., satellite or even some types of wireless cellular networks), also in comparison to widespread commercial solutions [5]. Moreover, the full functionalities of the system may be accessed from a wireless network in general, provided that a transmission speed in the range $0.8-1 \mathrm{Mbit} / \mathrm{s}$ is achievable. Problems regarding security should be handled by appropriate authentication and data protection. Possible QoS provision mechanisms may be adopted over the wireless link and at the wired/wireless network boundaries.

As regards specifically user mobility, a link with the mechanisms developed within the VICOM project (MIE - Mobile Immersive Environment - test-bed) for localization and user guidance may be established. Such mechanisms, based on the use of multiple localization techniques, would facilitate the mobile users in reaching specially equipped classrooms, where they can take advantage of advanced interfaces (e.g., multimedia board, haptic interfaces, or 3D video rendering).

Future developments will regard the establishment of a software interface between LIME (Linda In a Mobile Environment) [6], the middleware used for hiding the distribution of the context data in the MIE test-bed, and the VIL Database, to automatically acquire profiles of mobile users when they cross the classroom threshold.

\section{CONCLUSIONS}

The paper has presented the design and implementation of the VIL test-bed and its main related motivations, as well as critical aspects. The software and hardware strategies, allowing reproduce the context of a real academic classroom in a virtual environment, have been described in some detail.

High portability, good flexibility, and, above all, low cost, make this approach appropriate for educational and training purposes, mainly concerning measurements on telecommunication systems, at universities and research centers, as well as enterprises. Moreover, the methodology can be employed for remote access to and sharing of costly measurement equipment in many different fields of activity.

\section{ACKNOWLEDGMENTS}

This work was funded by the Italian Ministry of Education and Scientific Research (MIUR) in the framework of the FIRB_VICOM project.

\section{REFERENCES}

[1] Tougaw, D., Will, J., Visualizing the Future of Virtual Reality, Computing in Science \& Engineering, vol. 5, no. 4, pp. 8-11, July/August 2003.

[2] Pierleoni, P., Cancellieri, G., Biondi, F., Di Biase, T., Fioretti F., The Dynamic Construction of Multi-user VRML 3D Environment for Immersive Learning on the Web, in F. Davoli, S. Palazzo, S. Zappatore, Eds., Distributed Cooperative Laboratories: Networking, Instrumentation, and Measurements, Springer, New York, NY, 2006, pp. 497-509.

[3] Davoli, F., Spanò, G., Vignola, S., Zappatore, S., LABNET: towards remote laboratories with unified access, IEEE Transactions on Instrumentation and Measurement, vol. 55, no. 5, pp. 1551-1558, Oct. 2006.

[4] Vollono, A., Zinicola, A., A New Perspective in Instrumentation Interfaces as Web Ser-vices, in F. Davoli, S. Palazzo, S. Zappatore, Eds., Distributed Cooperative Laboratories: Networking, Instrumentation, and Measurements, Springer, New York, NY, 2006, pp. 451-461.

[5] Berruti L., Davoli F., Vignola S., Zappatore S., Interconnection of Laboratory Equipment via Satellite and Space Links: Investigating the Performance of Software Platforms for the Management of Measurement Instrumentation, Proc. 2006 International Tyrrhenian Workshop on Digital Communications, Ponza, Italy, Sept. 2006 (to appear).

[6] Picco G., Murphy A., Roman G., Developing Mobile Computing Applications with Lime, Proc. of the 22th International Conference on Software Engineering (ICSE 2000), Limerick (Ireland), M. Jazayeri and A. Wolf, eds., ACM Press, June 2000, pp. 766-769. 